\title{
Trust me, I'm a doctor!
}

\author{
Werner Bauera, Christoph Hänggelib
}

${ }^{a}$ Dr. med., Präsident des SIWF; ${ }^{b}$ Rechtsanwalt, Geschäftsführer des SIWF
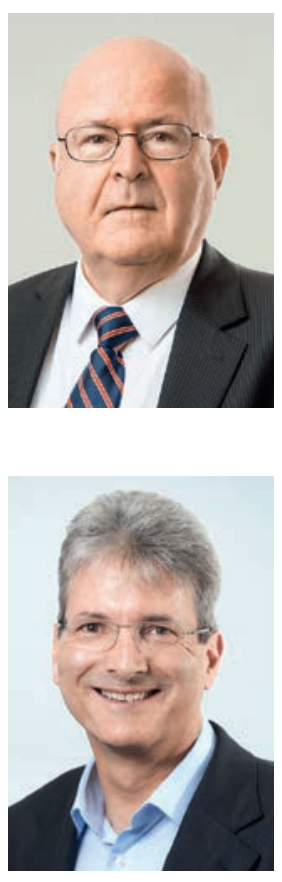

Die Plenarversammlung des Schweizerischen Instituts für ärztliche Weiter- und Fortbildung SIWF ist das oberste Beschlussorgan des Instituts und eine wichtige Diskussionsplattform für aktuelle Bildungsprobleme. Ein Teil des Plenums 2017, über das in dieser Ausgabe (S. 130) berichtet wird, war der Fortbildung gewidmet. Diese ist eine unbestreitbare Notwendigkeit für die Ärzteschaft, gibt aber hinsichtlich Inhalt, Umfang und Nachweis immer wieder Anlass zu Diskussionen. Offene Fragen und Kritikpunkte sind zum Beispiel:

- Die Fortbildung soll in Selbstverantwortung geschehen. Das Sammeln von Credits ist «Erbsenzählerei»!

- Die Fortbildung darf nicht in den Sog von «Reglementiererei» und "Kontrolliererei» geraten!

- Weiterentwickelte und neue Methoden, wie wir sie heute in den verschiedensten Fachbereichen beobachten, machen das Erwerben zusätzlicher Fähigkeiten im Laufe eines Arztlebens immer wichtiger. Man spricht im Angelsächsischen deshalb immer mehr von "continuing professional development» statt von "education».

- Die Fortbildung muss in Zukunft eine berufliche Weiterentwicklung und den Erwerb neuer Kompetenzen ermöglichen, die bei Abschluss der Weiterbildung noch nicht benötigt wurden.

- In bestimmten Fachgebieten können Empfehlungen zu den Lernzielen und das Angebot strukturierter Fortbildungszyklen sinnvoll sein.

- Die Fortbildung ist nicht reine Privatsache. Sie muss von der Ärzteschaft gegenüber den Patienten und der Öffentlichkeit glaubhaft dokumentiert werden.

Die Diskussion galt zunächst der Frage, inwieweit die Fortbildung individuell und frei gestaltet werden soll und inwieweit sie einer gewissen Strukturierung von Seiten der Fachgesellschaften bedarf. Besonders lebhaft und kontrovers wurden der Umfang der Doku-

\section{Die Fortbildung ist eine unbestreitbare Notwendigkeit für die Ärzteschaft.}

mentation und die Notwendigkeit einer Kontrolle besprochen. Ausgangspunkt war die Feststellung in der Mai-Nummer 2015 des JAMA: Individual physicians can no longer assert authority with complete autonomy and independence - getting by with «Trust me, I'm a doctor».
Das Meinungsspektrum reichte vom absoluten Primat der Selbstverantwortung und dem Vermeiden von vorauseilendem Gehorsam bis zur Einsicht, dass die Ärzteschaft nicht erst dann mit einer Selbstregulierung und verbindlichen Strukturierung reagieren sollte, wenn es gar nicht mehr anders geht. Tatsächlich bewahren wir uns letztlich mehr Freiheit, wenn wir selber die Fortbildung auch gegen aussen glaubhaft gestalten und sinnvoll dokumentieren - und zwar proaktiv.

Mit diesem Ziel bietet das SIWF eine browsergestützte Fortbildungsplattform an, auf der die Fortbildungsaktivitäten in einem persönlichen Protokoll erfasst werden können. Das Fortbildungsdiplom lässt sich nach Erreichen von 150 Credits selbständig ausdrucken - soweit alle Voraussetzungen des gewählten Fortbildungsprogramms erfüllt sind. Das jeweils für

\section{Alle 45 Fachgesellschaften haben sich der Fortbildungsplattform angeschlossen.}

eine Dreijahresperiode gültige Diplom ist ein MUSS für alle fortbildungspflichtigen Ärztinnen und Ärzte, weil damit nicht nur die gesetzlich vorgeschriebene Fortbildung nachgewiesen ist, sondern auch alle Besitzstandpositionen automatisch validiert werden. Inzwischen haben sich alle 45 Fachgesellschaften der Fortbildungsplattform angeschlossen, was ihnen mit minimalem Aufwand die gewünschte Kontrolle für die Erteilung der Diplome erlaubt.

Die Plenarversammlung hat nicht nur Grundsätze diskutiert, sondern auch zu administrativen Einzelfragen Stellung bezogen:

- Auch wer mehrere Facharzttitel besitzt, muss gemäss Fortbildungsordnung des SIWF nur ein Fortbildungsdiplom erwerben.

- Das Fortbildungsdiplom soll aus Kostengründen nur noch alle drei Jahre ausgestellt und ausgedruckt werden können.

- Alle Ärztinnen und Ärzte erhalten sechs Monate vor Ablauf der dreijährigen Gültigkeit ihres Fortbildungsdiploms automatisch ein Erinnerungsmail zur Erneuerung ihres Diploms.

Was wir mit sinnvoller Fortbildung und wenig Administration erreichen wollen, ist, weiterhin glaubhaft sagen zu können: Trust me, I'm a doctor. 